\title{
Evaluation of the Expression and Protective Potential of Leptospiral Sphingomyelinases
}

\author{
Eneas Carvalho $\cdot$ Angela S. Barbosa $\cdot$ Ricardo M. Gómez • \\ Maria L. S. Oliveira · Eliete C. Romero • Amane P. Gonçales • \\ Zenaide M. Morais $\cdot$ Sílvio A. Vasconcellos $\cdot$ Paulo L. Ho
}

Received: 27 May 2009/Accepted: 24 September 2009/Published online: 14 October 2009

(C) Springer Science+Business Media, LLC 2009

\begin{abstract}
Leptospirosis is a zoonotic disease of global distribution, which affects both animals and humans. Pathogenic leptospires, the bacteria that cause this disease, require iron for their growth, and these spirochetes probably use their hemolysins, such as the sphingomyelinases, as a way to obtain this important nutrient from host red blood cells during infection. We expressed and purified the leptospiral sphingomyelinases Sph1, Sph2, Sph4, and SphH in a heterologous system. However, the recombinant proteins were not able to lyse sheep erythrocytes, despite having regular secondary structures. Transcripts for all sphingomyelinases tested were detected by RT-PCR analyses, but only Sph2 and $\mathrm{SphH}$ native proteins could be detected in Western blot assays using Leptospira whole extracts as well as in renal tubules of infected hamsters. Moreover, antibodies present in the serum of a human patient with laboratory-confirmed leptospirosis recognized $\mathrm{Sph} 2$, indicating
\end{abstract}

Electronic supplementary material The online version of this article (doi:10.1007/s00284-009-9519-3) contains supplementary material, which is available to authorized users.

E. Carvalho · M. L. S. Oliveira · P. L. Ho ( $\square)$

Centro de Biotecnologia, Instituto Butantan, 05503-900 São

Paulo, SP, Brazil

e-mail: hoplee@butantan.gov.br

E. Carvalho $\cdot$ P. L. Ho

Departamento de Genética e Biologia Evolutiva, Instituto de Biociências, Universidade de São Paulo, São Paulo, SP, Brazil

E. Carvalho

Laboratório de Parasitologia, Instituto Butantan, São Paulo, SP, Brazil

\section{A. S. Barbosa}

Laboratório de Bacteriologia, Instituto Butantan, São Paulo, SP, Brazil that this sphingomyelinase is expressed and exposed to the immune system during infection in humans. However, in an animal challenge model, none of the sphingomyelinases tested conferred protection against leptospirosis.

\section{Introduction}

Pathogenic bacteria from the genus Leptospira can infect humans and almost all mammals, as well as reptiles and amphibians [5, 21]. Infection by Leptospira causes diverse damage in humans as a consequence of virulent factors produced by the bacteria [13]. Hemolysins are important virulent factors which are supposed to be responsible for erythrocyte lysis in the host, but their role in the pathogenic mechanism of the infection is still unclear [2, 28, 33]. After the sequencing of Leptospira genomes, several genes coding for predicted hemolysins were identified. The number of predicted hemolysins in Leptospira interrogans serovars Lai and Copenhageni is 10 or 11, respectively, and they can be grouped into sphingomyelinase hemolysins and

\author{
R. M. Gómez \\ Instituto de Biotecnología y Biología Molecular, Universidad \\ Nacional de La Plata, Centro Científico Tecnológico CONICET, \\ La Plata, Argentina \\ E. C. Romero \\ Seção de Bacteriologia, Instituto Adolfo Lutz, São Paulo, SP, \\ Brazil
}

A. P. Gonçales · Z. M. Morais · S. A. Vasconcellos

Faculdade de Medicina Veterinária e Zootecnia, Universidade de São Paulo, São Paulo, SP, Brazil 
non-sphingomyelinase hemolysins [18, 24, 33], where the sphingomyelinases are very similar in their amino acid composition and domain organization [33]. The sphingomyelinases are only found in pathogenic leptospires, whereas the non-sphingomyelinase hemolysins are also present in the saprophyte Leptospira biflexa Patoc, suggesting a possible contribution of sphingomyelinases to leptospiral survival inside hosts $[15,20,27]$. Since iron may represent a limiting factor in the growth of various pathogenic bacteria, it is conceivable that these toxins play a role in the persistence of the infection $[4,15]$. It is also speculated that sphingomyelinases may be involved in the generation of free fatty acids as a source of carbon and energy [3, 26]. In addition, the cytotoxicity of two sphingomyelinases has been demonstrated [12, 34], indicating that these proteins may display other properties which are not related to nutritional requirements. In fact, sphingomyelinase Sph2 was able to damage lymphocytes and macrophages [34], which may help leptospiral defense against the host immune system.

Leptospirosis is considered the most disseminated zoonosis in the world, and is also a reemerging disease [13, 30]. Moreover, there are few vaccines for human use, and they are licensed only in the countries in which they are produced [17]. These vaccines are based on inactivated bacteria or bacterial membrane preparations, tend to cause collateral effects, and protect only against specific serovars $[9,17,31]$. Since sphingomyelinases seem to be important virulent factors that are essential for leptospiral growth in the host, we examined their expression in various cultured serovars and also during infection, as well as their protective potential in an animal challenge against leptospirosis.

\section{Materials and Methods}

\section{Leptospira Strains and Culture}

Strains were obtained from the Laboratório de Zoonoses Bacterianas, FMVZ, Universidade de São Paulo, and were cultured in EMJH media supplemented with rabbit serum, as described in [8]. Virulence of one strain of L. interrogans serovar Pomona, namely Fromm, was maintained by iterative passages in Golden Syrian hamsters.

DNA Isolation and PCR analysis

Leptospira cultures were harvested by centrifugation at $5400 \times g$ for $30 \mathrm{~min}$ and gently washed twice in sterile PBS. Genomic DNA was isolated from the pellets with a guanidine-detergent lysing solution $\left(\right.$ DNAzol $^{\circledR}$ Reagent, Invitrogen, Carlsbad, CA), according to the manufacturer's instructions. The predicted coding sequences were amplified using the primers listed in Supplementary Table 1, designed according to $L$. interrogans serovar Copenhageni genome sequences (GenBank, accession AE016823).

\section{RNA Extraction and RT-PCR Analysis}

Leptospires were cultured at three different temperatures $\left(20,29\right.$, and $\left.37^{\circ} \mathrm{C}\right)$ and then harvested by centrifugation at $5400 \times g$ for $30 \mathrm{~min}$. For RT-PCR, total RNA was isolated by the acid guanidinium thiocyanate phenol-chloroform method using TRIzol ${ }^{\circledR}$ Reagent (Invitrogen) according to the manufacturer's recommendations. One microgram of RNA from each sample was treated with $1 \mathrm{U}$ of DNAse I (Invitrogen) for $15 \mathrm{~min}$ at room temperature. DNAse I was inactivated by the addition of $1 \mu \mathrm{l}$ of $25 \mathrm{mM}$ EDTA solution followed by incubation at $65^{\circ} \mathrm{C}$ for $10 \mathrm{~min}$. DNAse-treated RNAs were reversely transcribed using the SuperScript $^{\text {TM }}$ III First-Strand Synthesis System for RTPCR (Invitrogen). One-tenth of RT products was amplified using the oligonucleotides described in Supplementary Table 1. For the amplifications, we used 30 denaturingannealing-extension cycles.

Cloning, Expression, and Purification of Recombinant Proteins

The predicted coding sequences were amplified from DNA of L. interrogans serovar Copenhageni Fiocruz L1-130 (ATCC number BAA-1198), using the primers presented in Supplementary Table 1 . The coding DNA fragments exclude the sequence predicted to be signal peptides. The PCR product of each gene was cloned in $\mathrm{pAE}$, a vector with a promoter derived from $\mathrm{T} 7$ phage that adds an $\mathrm{N}$-terminal hexa-histidine tag to the recombinant protein [23]. Correct insertions were confirmed by sequencing. Escherichia coli BL21(DE3) Star pLysS (Novagen, Madison, WI) was transformed with each construct. Selected clones, expressing the recombinant proteins, were grown in 2YT medium until they reached an absorbance of 0.6 at $600 \mathrm{~nm}$ and then induced by the addition of $1.2 \mathrm{mM}$ of IPTG. The culture lysate was divided into supernatant and inclusion body precipitate by centrifugation. The inclusion bodies were dissolved in $8 \mathrm{M}$ urea, and then used for protein purification by nickel affinity chromatography, using standard protocols [25]. After purification, the proteins were extensively dialyzed against PBS $1 \times$ for urea removal before protein assays.

Antisera Against Recombinant Proteins

The antisera were produced in BALB/c mice, by intraperitoneal injection of $10 \mu \mathrm{g}$ of each recombinant protein 
and $100 \mu \mathrm{g}$ of aluminum ion (added in the form of $\left.\mathrm{Al}(\mathrm{OH})_{3}\right)$ every week for 4 weeks. Antibody titers were determined by ELISA, using $1 \mu \mathrm{g}$ of the recombinant proteins as coating per well. Absorbance was read at $492 \mathrm{~nm}$, and the serum dilution with a reading of 0.1 was considered the serum titer. Human anti-Leptospira sera were obtained from convalescent patients with laboratoryconfirmed leptospirosis; the sera were collected in the second week after symptoms had appeared, and their titers were estimated by the microagglutination test, as described by Faine et al. [5].

\section{Western Blot and Immunoprecipitation Assays}

Samples were submitted to SDS-PAGE and then transferred to a nitrocellulose membrane for Western blotting. The membrane was blocked with $10 \%$ (wt/vol.) skim milk overnight at $4^{\circ} \mathrm{C}$, and then incubated with the serum of interest for $90 \mathrm{~min}$. After three washes of $10 \mathrm{~min}$ with $0.05 \%$ (v/v) Tween20 in PBS (PBS-T), the membrane was incubated with an appropriate antibody-peroxidase conjugate for $60 \mathrm{~min}$. After three washes of $10 \mathrm{~min}$ with PBS-T, the membrane was revealed with ECL reagent (GE Healthcare, Waukesha, WI). Alternatively, sphingomyelinases were immunoprecipitated from culture supernatant essentially as described by Matsunaga et al. [16], including a cleaning step of the samples with a previous incubation with protein $\mathrm{A}$ immobilized in agarose beads, in order to remove immunoglobulins from the rabbit serum used for leptospire culture.

\section{Detection of Leptospiral Protein Expression In Vivo}

Hamsters were inoculated intraperitoneally with $0.5 \mathrm{ml}$ of PBS containing $10^{2.5}$ bacteria ( $L$. interrogans serovar Icterohaemorrhagiae), sacrificed 2 weeks post-infection when animals showed symptoms (e.g., weight loss, lethargy), and their kidneys were harvested and processed for routine immunohistochemical analyses. Samples of kidney tissue were incubated with the antisera generated against rSph2 and $\mathrm{rSphH}$. For detection of antibody-antigen complexes, we used the colorimetric system Envision (DAKO), according to the manufacturer's instructions. Mock infected animals were used as controls.

\section{Circular Dichroism}

The presence of ordered secondary structures in the recombinant proteins was assessed by circular dichroism (CD) spectroscopy in a JASCO J-810 spectropolarimeter. Proteins were previously dialyzed in $20 \mathrm{mM}$ sodium

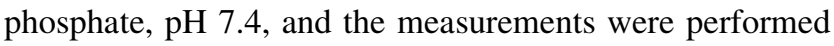
from 190 to $260 \mathrm{~nm}$, at intervals of $0.5 \mathrm{~nm}$. The CD spectra presented are the average of five measurements.
Hemolytic Assays

These assays were performed as described elsewhere [2]. As a positive control, $0.8 \mu \mathrm{g}$ of sphingomyelinase $\mathrm{C}$ from Staphylococcus aureus (Sigma, St. Louis, MO) was used. Erythrocytes incubated with a non-hemolytic recombinant protein from Schistosoma mansoni (fatty acid binding protein Sm14) [22] were used as negative control. Additional controls were included by pre-incubation of each protein with PBS, or 1:20 dilutions of immune serum or non-immune serum. For hemolytic assays with L. interrogans Pomona Fromm and L. biflexa Patoc, we used $70 \mu \mathrm{l}$ (in a $100 \mu$ total reaction mix) of (a) the total culture of bacteria grown for 10 days, until they are close to saturation ( $\sim 10^{8}$ cells $/ \mathrm{ml}$ ), (b) bacteria washed with PBS, or (c) culture supernatants. Bacteria from groups (a) and (b) were lysed by vortexing ice-cooled samples for $10 \mathrm{~min}$ with glass beads. After incubation with the sheep erythrocytes, the reaction samples were centrifuged and read at $420 \mathrm{~nm}$. We also tested if the hemolysis produced by Leptospira could be inhibited by antisera. In this experiments, total cultures of Leptospira were pretreated with several sera (1:20) for $30 \mathrm{~min}$ before hemolytic assay. The leptospirosis patient sera used were a pool of seven individuals. All experiments were performed in triplicate and repeated three times.

\section{Challenge Experiments}

A group of ten hamsters was intradermally immunized twice with $50 \mu \mathrm{g}$ of $\mathrm{rSph} 2$ or $\mathrm{rSphH}$ plus $500 \mu \mathrm{g}$ of aluminum ion (added in the form of $\mathrm{Al}(\mathrm{OH})_{3}$ ), with a 21-day interval, which was a procedure modified from Palaniappan et al. [19]. On day 42, the hamsters were challenged with approximately $1 \mathrm{LD}_{50}\left(6 \times 10^{3}\right)$ of $L$. interrogans serovar Pomona Fromm injected intraperitoneally. Twenty-one days after challenge (on day 63), the surviving hamsters were killed and their kidneys were removed and tested for
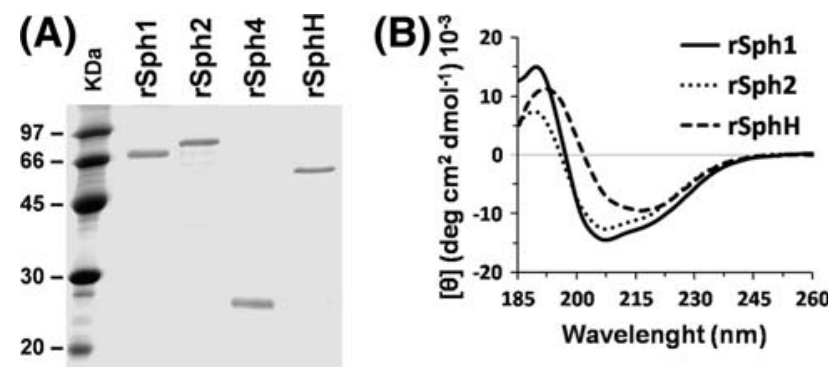

Fig. 1 Purified recombinant proteins and their CD spectra. a SDSPAGE of the purified recombinant sphingomyelinases, stained with Coomassie blue; $\mathbf{b}$ the pattern of the $\mathrm{CD}$ spectra indicates that the refolded recombinant sphingomyelinases have regular secondary structures. rSph4 was diluted in a chaotropic solution and did not have regular secondary structures. The spectra shown are the average of five measurements 
Fig. 2 Evaluation of the hemolytic ability of the recombinant sphingomyelinases and of leptospires. a Hemolytic tests show that the refolded recombinant sphingomyelinases do not exhibit hemolytic activity. Sheep erythrocytes were incubated with the recombinant refolded proteins and with sphingomyelinase $\mathrm{C}$ from $S$. aureus as the positive control. Leptospiral (L. interrogans serovar Pomona, strain Fromm) cell lysate and culture supernatant were included as additional controls. Each sample group was pre-treated with PBS, specific antiserum (except for leptospiral cell lysate and culture supernatant and $S$. aureus groups), or non-immune serum. The asterisks indicate statistical significance at $\alpha=0.01$ in a TukeyKramer HSD test. b Pathogenic Leptospira were capable of hemolysis, whereas saprophyte Leptospira were not. Sheep erythrocytes were incubated with Leptospira culture, with culture supernatant or with cell lysate. All the hemolytic activities of Leptospira were present in the culture supernatant. The asterisks indicate statistical significance at $\alpha=0.01$ in a Tukey-Kramer HSD test. c Leptospira hemolytic activity was similarly reduced by all tested sera, even by non-immune serum. The cultures of leptospires were pre-treated with sera (1:20) and then incubated with sheep erythrocytes. All sample means were statistically different from the control PBS group at $\alpha=0.01$ in a Tukey-Kramer HSD test. Total hemolysis was obtained by incubating the sheep erythrocytes with distilled water. All the experiments were carried out in triplicate and repeated three times

the presence of Leptospira by culture in EMJH medium. The positive control group was immunized with the commercial vaccine (FarrowSure ${ }^{\circledR}$ B, Pfizer, New York, NY) and the negative control group with PBS. Hamsters were bled before the immunization (day 0), 1 day before the challenge (day 41) and at the time of killing (day 63). Antibody titers were determined as described above.

\section{Results}

The Recombinant Sphingomyelinases Have Regular Secondary Structures, But They Do Not Show Hemolytic Activity

The expression and purification of recombinant Sph1, Sph2, $\mathrm{Sph} 4$, and SphH were successfully achieved and the CD spectra indicate that these recombinant sphingomyelinases have regular secondary structures after the refolding process (Fig. 1), with the exception of Sph4. A number of standard refolding protocols were tested for this particular protein, but proper refolding could not be achieved since it precipitates in non-chaotropic solutions. Therefore, Sph4 was used exclusively in the Western blotting assays. Sph3 could not be expressed using the system described above. An assay with sheep erythrocytes was used to measure the hemolytic activity of the recombinant proteins. Whereas the positive control (sphingomyelinase from $S$. aureus, Sigma) was fully able to lyse sheep erythrocytes, none of the recombinant leptospiral sphingomyelinases (Sph1, Sph2, and $\mathrm{SphH}$ ) could produce hemolysis, since OD values did not differ from those shown by the negative control groups (PBS and the non-hemolytic recombinant protein Sm14, a
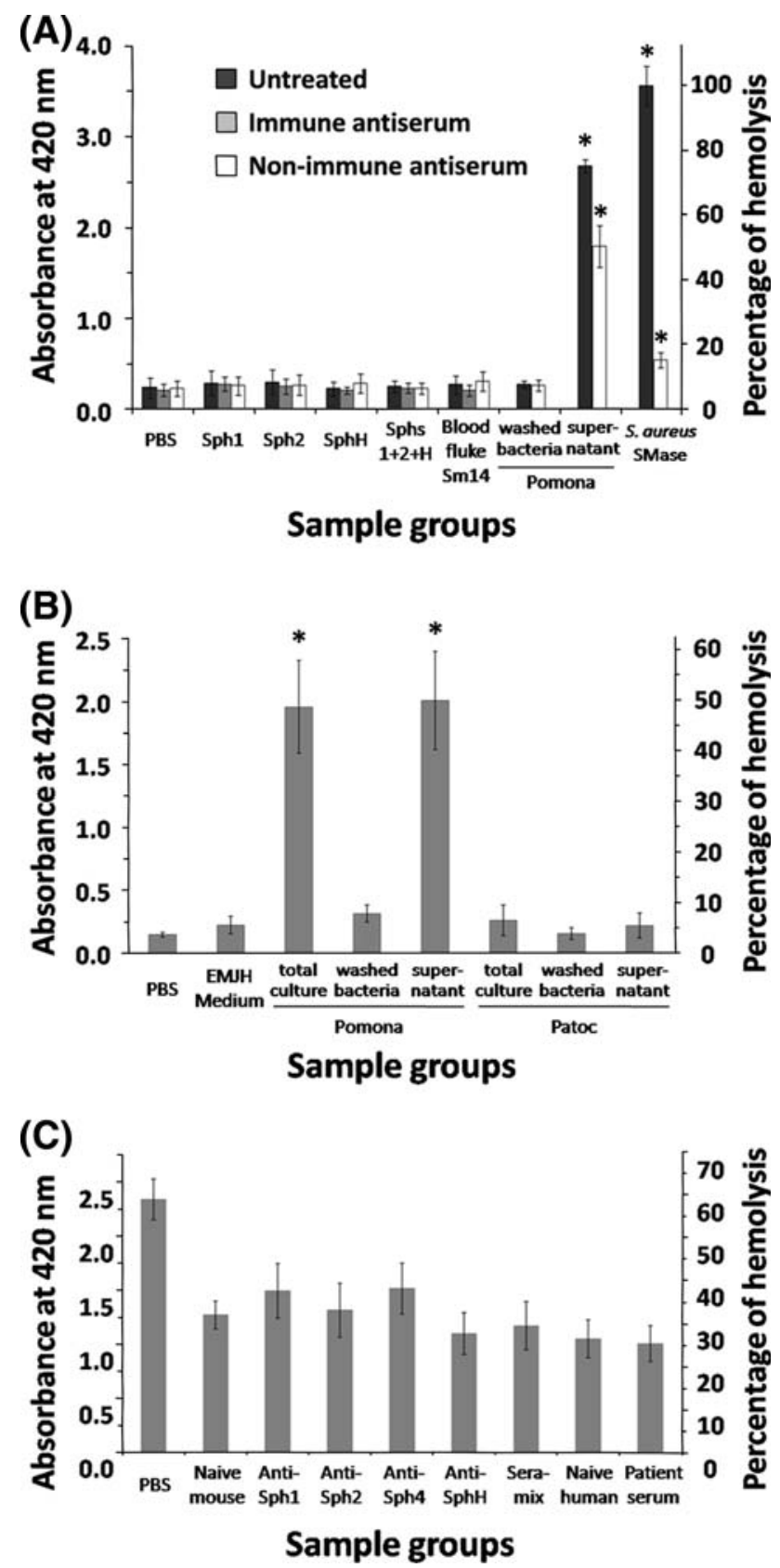

fatty acid-binding protein from the blood fluke helminth $S$. mansoni) (Fig. 2a).

Pathogenic Leptospires Secrete Hemolytically Active Factors That Are Inhibited by Immune and Non-Immune Sera

A virulent strain of the pathogenic L. interrogans serovar Pomona was able to produce hemolysis, whereas a strain of the saprophytic species L. biflexa serovar Patoc was not (Fig. 2b). Our data indicate that the hemolytic factors produced by leptospires are secreted, since the hemolytic 
activity observed in the total culture lysate was not distinguishable from that observed in the culture supernatant. Conversely, the lysate of washed leptospires was unable to produce hemolysis. Curiously, the hemolytic activity observed in pathogenic leptospire cultures was non-specifically inhibited by the addition of any serum (Fig. 2c). As shown in Fig. 2c, sera from leptospirosis patients and from a healthy person produced a similar degree of inhibition of the hemolytic activity of leptospires. Similarly, the percentage of hemolysis obtained with serum from mice immunized with recombinant sphingomyelinases did not differ from that observed with serum from naive mice (Fig. 2c). This kind of inhibition was also observed for the positive control $S$. aureus sphingomyelinase (Fig. 2a).

Leptospiral Sphingomyelinases Are Expressed

in Culture, In Vivo, and During Infection in Hamsters and in Humans

Sph1, Sph2, Sph3, Sph4, and SphH transcripts were detected in cultured $L$. interrogans serovar Pomona grown in three different temperatures $\left(20,29\right.$, and $\left.37^{\circ} \mathrm{C}\right)$ (Fig. 3a). Nevertheless, when analyzing the leptospiral lysates, protein expression was only observed for $\mathrm{Sph} 2$ and $\mathrm{SphH}$, and it occurred exclusively in L. interrogans serovar Pomona (both in low and high passages) (Fig. 3b). The expression of both proteins in cell lysates was detected in early log and in stationary growth phase (Fig. 4b, d, cell lysate), although Sph2 was also detected in culture supernatant, most of them in the early log phase (Fig. 4b, culture supernatant, lanes 3 and 5). A precise detection of SphH in the culture supernatant was not possible due to the presence of immunoglobulin heavy chain in the same molecular mass range of $\mathrm{SphH}$, recognized by the secondary anti-IgG mouse peroxidase conjugate. Although anti-Sph2 and anti$\mathrm{SphH}$ cross reacted with $\mathrm{rSph} 2$ and $\mathrm{rSphH}$ (Fig. 3b), the antiserum produced against $\mathrm{rSph} 2$ was able to cross react with a leptospiral protein compatible with the expected molecular mass of native $\mathrm{SphH}$, whereas the antiserum against $\mathrm{rSphH}$ only cross reacted with the recombinant Sph2 (Fig. 4b, d) as previously shown (Fig. 3b). Protein expression of Sph1 and Sph4 was not detected in any serovar (data not shown) and Sph3 expression was not investigated. To evaluate the expression of $\mathrm{Sph} 2$ and $\mathrm{SphH}$ during animal infection, infected hamsters were killed and immunohistochemical analyses were carried out in their renal tissues (Fig. 5a, b). Intense signals were detected using anti-Sph2 and anti-SphH in hamster renal tubules, which represent important leptospire colonizing sites. The expression of the sphingomyelinases during human infection was also observed in immunoblot analyses. IgG antibodies present in the sera of patients with leptospirosis recognized only Sph2 (Fig. 5c), indicating that this protein

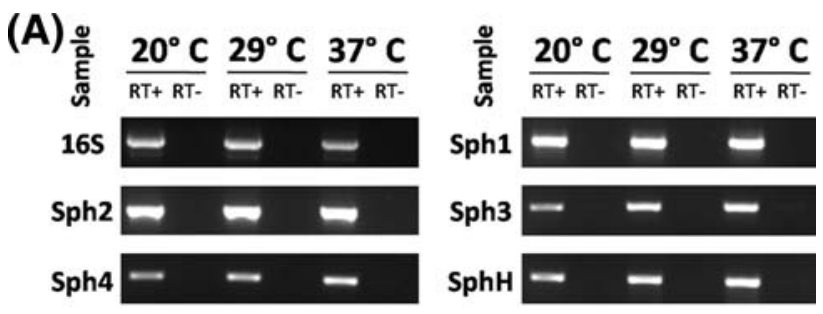

(B)

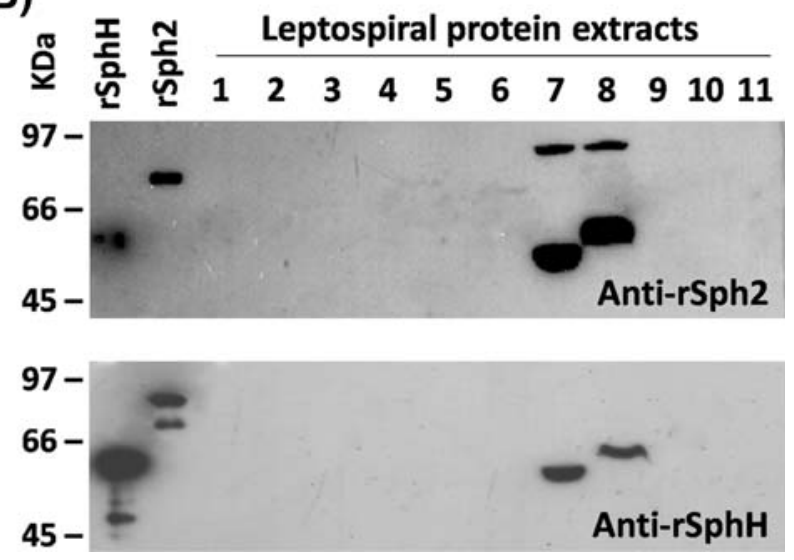

Fig. 3 Transcription and expression of the sphingomyelinases, in culture conditions. a Detection of gene transcription by RT-PCR. The transcriptions of $s p h 1, s p h 2, s p h 3, s p h 4$, and $s p h H$ are shown (RT+) in different growth conditions $\left(20,29\right.$, and $\left.37^{\circ} \mathrm{C}\right)$. A negative control (RT-), without reverse transcriptase, was used in each case. The amplification of a $16 \mathrm{~S}$ ribosomal RNA fragment was used as a control of cDNA integrity. This experiment was performed using the $L$. interrogans serovar Pomona strain Fromm. b The expression of the native proteins $\mathrm{Sph} 2$ and $\mathrm{SphH}$ by Western blot analyses in a panel of several Leptospira serovar extracts is showed. Both antisera cross react with the other recombinant sphingomyelinase, but just anti-Sph2 cross reacts with native proteins. Lanes (serovar and strain are, respectively, shown after leptospire species): 1 L. bifexa Patoc Patoc I, 2 L. interrogans Hardjo Hardjoprajitno, 3 L. interrogans Autunalis Akiyami A, 4 L. interrogans Pyrogenes Salinem, 5 L. interrogans Icterohaemorraghiae RGA, 6 L. interrogans Copenhageni M20, 7 L. interrogans Pomona Pomona (high passages), $8 \mathrm{~L}$. interrogans Pomona Fromm (low passages), 9 L. interrogans Bratislava Jez Bratislava, $10 \mathrm{~L}$ interrogans Canicola Hond-UtrechtIV, $11 \mathrm{~L}$. kirshneri Grippothyphosa MoskvaV

is expressed and presented to the host immune system. Antibodies against the positive control LipL32 (the major leptospiral outer membrane protein) [2, 7] were also detected, whereas no detection was observed with the negative control rSm14.

Sph2 and SphH Were Not Protective in an Animal Challenge Against Leptospirosis

Sph2 or SphH-immunized hamsters were challenged with a virulent strain of $L$. interrogans serovar Pomona (Fig. 6a). A group of hamsters was also immunized with a positive control (a commercial bacterin vaccine against leptospirosis) 

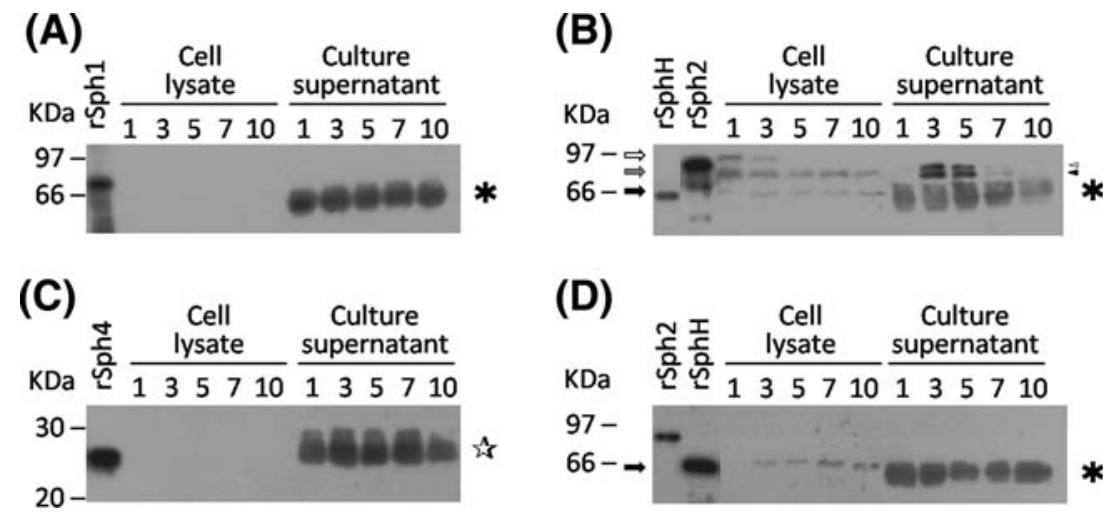

Fig. 4 Evaluation of sphingomyelinases expression during leptospiral growth phases, in cell lysate and culture supernatant. The antiserum used for protein detection in each figure was a anti-Sph1, b anti-Sph2, c anti-Sph4, d anti-SphH. The numbers under bars indicate the number of days in culture. Arrows and arrows head indicate possible sphingomyelinases produced by leptospires. White arrow Sph2 cell-associated heavy band; gray arrow Sph2 cell-associated light band; black arrow $\mathrm{SphH}$ band; white arrow head $\mathrm{Sph} 2$ secreted

and a negative control group with saline (PBS). The mortality of the hamsters immunized with the recombinant $\mathrm{Sph} 2$ and $\mathrm{SphH}$ was similar to that observed in animals immunized with PBS. On the other hand, the commercial vaccine conferred full protection. The immunization with both recombinant proteins was able to raise specific antibodies in hamster sera, as detected by ELISA (Fig. 6b). Hamster sera did not have detectable antibodies against both $\mathrm{rSph} 2$ and $\mathrm{rSphH}$ prior to the immunization (Fig. 6b). None of the surviving animals that were immunized with the commercial vaccine showed leptospires in their kidneys, whereas all the surviving animals immunized with PBS or the recombinant proteins did.

\section{Discussion}

Sphingomyelinases are proteins present in both eukaryotes and prokaryotes; while they are related to phospholipid metabolism in the former, in the latter they are usually not necessary to the life cycle of the organism and frequently act as toxins [6]. As pointed by Picardeau et al. [20], the role that sphingomyelinases play in Leptospira is still unclear. However, some reports demonstrating that Sph2 and $\mathrm{SphH}$ have hemolytic and cytotoxic activities, indicating that sphingomyelinases may have important functions in leptospiral pathogenesis [12, 34]. In an apparent contradiction, the recombinant proteins that we purified were not able to lyse sheep erythrocytes. A possible explanation for this absence of hemolytic activity lies in the fact that all proteins used in this study were expressed as inclusion bodies, and then subjected to refolding. Lack or reduced $(\sim 6 \%)$ hemolytic activity displayed by recombinant

heavy band; dark arrow head Sph2 secreted light band. Black stars indicate the reaction of anti-mouse IgG-peroxidase with mouse immunoglobulin heavy chain. White star indicates the reaction with the immunoglobulin light chain. Control recombinant proteins were shown in each figure. The cell lysate in each lane had $10^{8}$ leptospires. The density of leptospires (in bacteria per $\mathrm{ml}$ ) in each day was day $0=4.83 \times 10^{7}$, day $1=1.21 \times 10^{8}$, day $3=4.31 \times 10^{8}$, day $5=4.81 \times 10^{8}$, day $7=5.81 \times 10^{8}$, and day $10=5.58 \times 10^{8}$

sphingomyelinases expressed as inclusion bodies has already been reported by other authors $[1,11]$. Conversely, when these recombinant proteins are expressed as soluble proteins, their activity is preserved, reaching efficiencies of $100 \%$ hemolysis $[12,34]$. Thus, it seems that the sphingomyelinase structures obtained after refolding may not fully resemble the final native conformation. Several attempts were made to obtain soluble recombinant proteins, such as expression in various $E$. coli hosts and growth conditions (e.g., expression at $25^{\circ} \mathrm{C}$, lower concentration of the inducer, shorter induction times), but none of them was efficient in producing soluble proteins (data not shown). The use of a fusion partner or the expression with a tight promoter would make more probable the expression of soluble recombinant sphingomyelinases.

The ability to produce hemolysis is present in a pathogenic serovar (L. interrogans Pomona), but absent in a saprophytic one (L. biflexa Patoc). Moreover, this activity is confined to the culture medium, indicating that the leptospiral hemolytic factors are secreted. These observations are in accordance with the possible involvement of sphingomyelinases in the hemolytic process, since only pathogenic serovars have sphingomyelinase genes [14, 27], and also because sphingomyelinase activity was detected in the supernatant fraction of cultures of the serovar Pomona [26].

Notably, the hemolytic activity of leptospires was inhibited by the addition of any sera. This phenomenon was first reported by Yanagihara et al. [32] and Kojima et al. [10], who also showed that the cause of such inhibition was a competition between the hemolysin targets (the phospholipids and sphingomyelins of the erythrocyte membrane) and the free phospholipids present in serum (especially with 
(A)
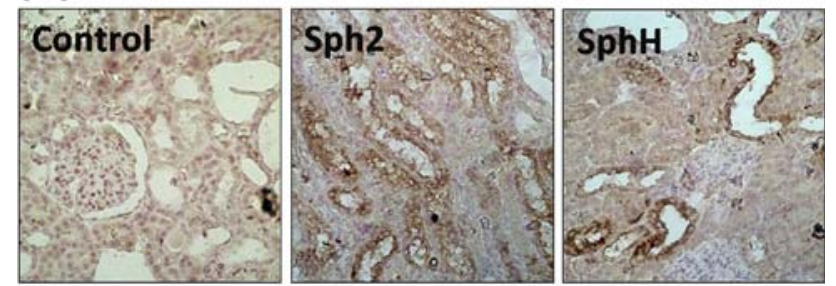

(B)

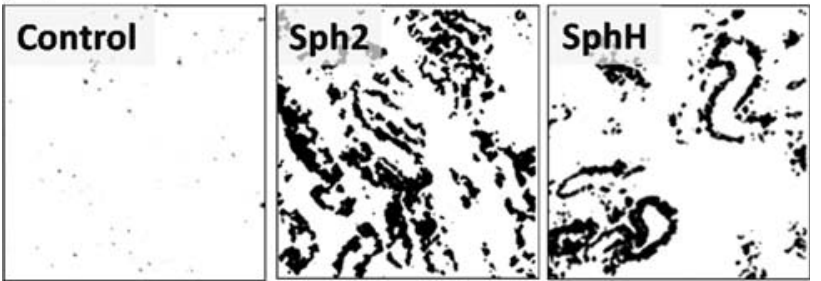

(C)

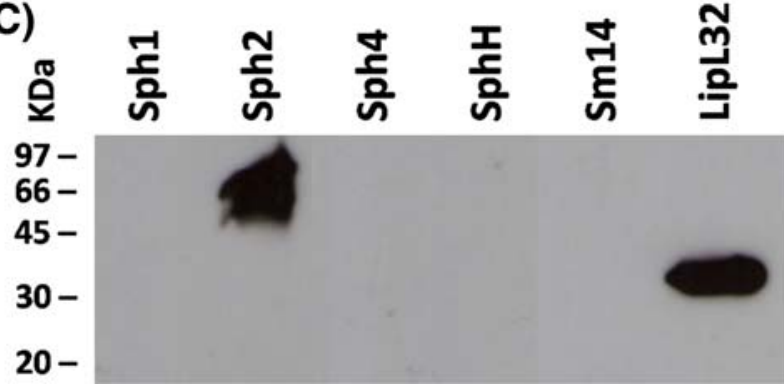

Fig. 5 Detection of leptospiral sphingomyelinases expression in infected hamsters and serological evidence of its expression in humans. a The immunohistochemical detections are shown using anti-Sph2 and anti-SphH, in the renal tubular epithelium of infected hamsters. Original magnification: $\times 250$. b The labeled renal tubular epithelium is evidenced in a shadow representation. $\mathbf{c}$ IgG antibodies present in serum from a patient with leptospirosis recognize the recombinant Sph2. Similar results were obtained using sera from other four patients. Each recombinant sphingomyelinase sample and rSm14 contain $2 \mu \mathrm{g}$ of protein; the positive control rLipL32 contains $0.5 \mu \mathrm{g}$

the sphingomyelins and phosphatidylcholines). We also observed the inhibitory effect of sera in the sphingomyelinase of $S$. aureus, suggesting that this phenomenon can be a common consequence of a competitive inhibition with serum components. Thompson and Marshall [29] hypothesized that the in vitro mechanism responsible for the lysis of erythrocytes in vitro is different from that which causes hemolysis during a leptospiral infection. However, unexpectedly, sera from leptospirosis patients and from mice immunized with recombinant sphingomyelinases were not able to produce a higher degree of inhibition than that observed using sera from naive mice or from healthy humans. Thus, the presence of antibodies against sphingomyelinases did not increase the inhibition of leptospiremediated hemolysis, possibly because the sphingomyelinases were already fully inhibited by the free phospholipids
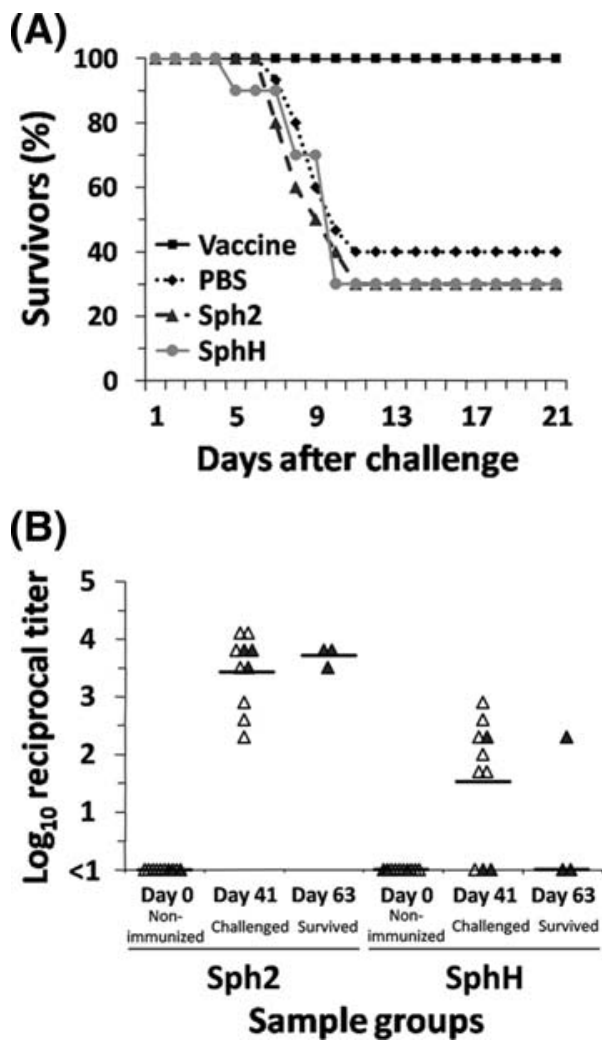

Fig. 6 Survival of immunized hamsters after challenge and serum titers. Ten hamsters were immunized with $\mathrm{rSph} 2, \mathrm{rSphH}$, saline, or a commercial vaccine and then challenged with $L$. interrogans serovar Pomona strain Fromm. a A survival plot shows that none of the recombinant proteins tested was protective in this animal challenge. $\mathbf{b}$ IgG antibodies against the recombinant proteins were detected in animals immunized with both $\mathrm{Sph} 2$ and $\mathrm{SphH}$, just prior to the challenge (day 41); antibodies were not detected in the PBS or vaccine groups (data not shown). Individual sera titers are represented by triangles (solid and open triangles represent surviving and dead animals, respectively). For each group, the solid line indicates the geometric mean titer. Surviving animals did not show a detectable reduction in their titer value after the challenge (see the solid triangles in day 41 and day 63). Before the immunization (day 0), there were no detectable levels of anti-Sph2 or SphH in hamsters serum. The titer was defined as the last dilution in which an absorbance of 0.1 could be observed. The individual sera that displayed undetectable antirecombinant protein $\mathrm{IgG}$ titers were represented as $<1$

present in the serum. On the other hand, although these phospholipids were able to inhibit sphingomyelinasemediated hemolysis, some level of hemolysis is retained after the treatment with sera, indicating the presence of other possible leptospiral hemolysins, not directly inhibited by sera.

Transcripts for all sphingomyelinases tested were detected in leptospires cultured in EMJH supplemented with rabbit serum. However, when analyzing the cell lysate of these bacteria grown in this condition, we only detected the expression of $\mathrm{Sph} 2$ and $\mathrm{SphH}$ in L. interrogans serovar Pomona (both in low and high passages). Similar to 
previous results described elsewhere [16], we observed the expression of Sph2 and SphH in leptospiral early log and stationary phase, with the expression of $\mathrm{Sph} 2$ being detected both in the cell lysate and in the culture supernatant, whereas the expression of the other sphingomyelinases was not detected in any condition (Fig. 4). AntiSph2 recognized two bands in the culture supernatant (Fig. 4b), similar to the results described by Matsunaga et al. [16], which suggest that they could represent different processed forms of the cell-associated Sph2. Moreover, we also observed two different bands in the cell lysate, in days 1 and 3, which may also be associated to distinct cell processed Sph2 protein (Fig. 4b). Matsunaga et al. [16] detected the expression of Sph2 in culture conditions, but only associated with conditions of high osmolarities. They suggested that the addition of rabbit serum in culture medium could increase the osmolarity of the medium sufficiently to raise $\mathrm{Sph} 2$ to detectable levels. They also found that Sph2 (detected in the cell lysate and in the culture supernatant) was regulated by the leptospiral growth phase. They detected a cross-reacting band in the cell lysate, which was suggested to be the SphH protein and which does not seem to be regulated by the growth phase. Although the sphingomyelinases may not be necessary for leptospiral growth in culture conditions, the osmolarity or some components from rabbit serum (used to supplement the culture medium) may resemble the host environment, triggering the transcription and translation of $\mathrm{Sph} 2$ and $\mathrm{SphH}$ sphingomyelinases detected in serovar Pomona. Apparently, the translation process requires more stringent conditions to take place, since we detected the transcription of Sph1, Sph2, Sph3, Sph4, and SphH, but only the translation of Sph2 and $\mathrm{SphH}$ (Sph3 was not assayed). Although it is expected that these proteins are secreted to the environment, their presence in the bacterial lysate may be associated with newly synthesized proteins and also with protein trafficking through the secretory pathway.

It is also likely that $\mathrm{Sph} 2$ and $\mathrm{SphH}$ are expressed in vivo during leptospiral infection, in hamster renal tubules (Fig. 5a, b). However, it is possible that only one of them was expressed, since we used cross-reacting antisera (Figs. 3 and 4). Matsunaga et al. [16] also detected cross recognition with $\mathrm{SphH}$ when using antiserum generated against Sph2. Additionally, IgG antibodies present in the serum of leptospirosis patients recognized $\mathrm{rSph} 2$. Therefore, although we do not know for sure why sphingomyelinases are expressed, these data suggest that at least some of them are expressed by the leptospires during infection, which is in accordance with their probable role in pathogenesis. Since we obtained non-active recombinant proteins, they could be harmlessly injected in hamsters, and thus tested as vaccinal antigens. Furthermore, we showed that antibodies generated against the recombinant sphingomyelinases are able to recognize the native proteins. However, whereas the leptospiral sphingomyelinases seem to be necessary for iron acquisition, are able to kill immune system cells and are expressed during infection, immunization with $\mathrm{rSph} 2$ and $\mathrm{rSphH}$ was not able to protect hamsters from mortality upon challenge with leptospires. Apparently, the cross-recognition observed among sera against the sphingomyelinases was not enough to completely abolish the vital benefits resulting from the hemolysin activities. The presence of multiple hemolysins in the leptospire genome may explain why the bacteria can overcome the presence of antibodies against some of their hemolysins.

In conclusion, we could not demonstrate that the sphingomyelinases are directly involved in the hemolytic capacity of leptospires. However, this can be related to a lack of correct folding of the recombinant proteins. On the other hand, we showed that leptospiral Sph2 and SphH are expressed in culture, in vivo, and during human infection, although these proteins were not protective in a challenge experiment against leptospirosis.

Acknowledgments We thank Dr. Albert Leyva for English editing of the manuscript and FAPESP, CNPq, and Fundação Butantan for financial support.

\section{References}

1. Artiushin S, Timoney JF, Nally J et al (2004) Host-inducible immunogenic sphingomyelinase-like protein, Lk73.5, of Leptospira interrogans. Infect Immun 72:742-749

2. Carvalho E, Barbosa AS, Gomez RM et al (2009) Leptospiral TlyC is an extracellular matrix-binding protein and does not present hemolysin activity. FEBS Lett 583:1381-1385

3. del Real G, Segers RP, van der Zeijst BA et al (1989) Cloning of a hemolysin gene from Leptospira interrogans serovar hardjo. Infect Immun 57:2588-2590

4. Faine S (1959) Iron as a growth requirement for pathogenic Leptospira. J Gen Microbiol 20:246-251

5. Faine S, Adler B, Bolin C et al (1999) Leptospira and leptospirosis, 2nd edn. MediSci, Melbourne, Australia

6. Goni FM, Alonso A (2002) Sphingomyelinases: enzymology and membrane activity. FEBS Lett 531:38-46

7. Haake DA, Chao G, Zuerner RL et al (2000) The leptospiral major outer membrane protein LipL32 is a lipoprotein expressed during mammalian infection. Infect Immun 68:2276-2285

8. Hauk P, Macedo F, Romero EC et al (2008) In LipL32, the major leptospiral lipoprotein, the $\mathrm{C}$ terminus is the primary immunogenic domain and mediates interaction with collagen IV and plasma fibronectin. Infect Immun 76:2642-2650

9. Koizumi N, Watanabe H (2005) Leptospirosis vaccines: past, present, and future. J Postgrad Med 51:210-214

10. Kojima T, Yanagihara Y, Mifuchi I (1984) Characterization of inhibitor to leptospiral hemolysin present in bovine serum. Microbiol Immunol 28:291-302

11. Lee SH, Kim KA, Park YG et al (2000) Identification and partial characterization of a novel hemolysin from Leptospira interrogans serovar lai. Gene 254:19-28 
12. Lee SH, Kim S, Park SC et al (2002) Cytotoxic activities of Leptospira interrogans hemolysin $\mathrm{SphH}$ as a pore-forming protein on mammalian cells. Infect Immun 70:315-322

13. Levett PN (2001) Leptospirosis. Clin Microbiol Rev 14:296-326

14. Louvel H, Saint Girons I, Picardeau M (2005) Isolation and characterization of FecA- and FeoB-mediated iron acquisition systems of the spirochete Leptospira biflexa by random insertional mutagenesis. J Bacteriol 187:3249-3254

15. Louvel H, Bommezzadri S, Zidane $\mathrm{N}$ et al (2006) Comparative and functional genomic analyses of iron transport and regulation in Leptospira spp. J Bacteriol 188:7893-7904

16. Matsunaga J, Medeiros MA, Sanchez Y et al (2007) Osmotic regulation of expression of two extracellular matrix-binding proteins and a haemolysin of Leptospira interrogans: differential effects on LigA and Sph2 extracellular release. Microbiology 153:3390-3398

17. McBride AJ, Athanazio DA, Reis MG et al (2005) Leptospirosis. Curr Opin Infect Dis 18:376-386

18. Nascimento AL, Ko AI, Martins EA et al (2004) Comparative genomics of two Leptospira interrogans serovars reveals novel insights into physiology and pathogenesis. J Bacteriol 186:21642172

19. Palaniappan RU, McDonough SP, Divers TJ et al (2006) Immunoprotection of recombinant leptospiral immunoglobulin-like protein A against Leptospira interrogans serovar Pomona infection. Infect Immun 74:1745-1750

20. Picardeau MD, Bulach DM, Bouchier C et al (2008) Genome sequence of the saprophyte Leptospira biflexa provides insights into the evolution of Leptospira and the pathogenesis of leptospirosis. PLoS ONE 3:e1607

21. Plank R, Dean D (2000) Overview of the epidemiology, microbiology, and pathogenesis of Leptospira spp. in humans. Microbes Infect 2:1265-1276

22. Ramos CR, Figueredo RC, Pertinhez TA et al (2003) Gene structure and M20T polymorphism of the Schistosoma mansoni Sm14 fatty acid-binding protein. Molecular, functional, and immunoprotection analysis. J Biol Chem 278:12745-12751
23. Ramos CR, Abreu PA, Nascimento AL et al (2004) A high-copy T7 Escherichia coli expression vector for the production of recombinant proteins with a minimal $\mathrm{N}$-terminal His-tagged fusion peptide. Braz J Med Biol Res 37:1103-1109

24. Ren SX, Fu G, Jiang XG et al (2003) Unique physiological and pathogenic features of Leptospira interrogans revealed by wholegenome sequencing. Nature 422:888-893

25. Sambrook J, Russell RW (2001) Molecular cloning: a laboratory manual, 3rd edn. Cold Spring Harbor Laboratory Press, Cold Spring Harbor, NY

26. Segers RP, van der Drift A, de Nijs A et al (1990) Molecular analysis of a sphingomyelinase $\mathrm{C}$ gene from Leptospira interrogans serovar hardjo. Infect Immun 58:2177-2185

27. Segers RP, van Gestel JA, van Eys GJ et al (1992) Presence of putative sphingomyelinase genes among members of the family Leptospiraceae. Infect Immun 60:1707-1710

28. Thompson JC, Manktelow BW (1986) Pathogenesis and red blood cell destruction in haemoglobinaemic leptospirosis. J Comp Pathol 96:529-540

29. Thompson JC, Marshall RB (1986) In vitro studies of haemolysis by Leptospira interrogans serovars pomona and ballum. Vet Microbiol 11:285-292

30. Vinetz JM (2001) Leptospirosis. Curr Opin Infect Dis 14:527538

31. Wang Z, Jin L, Wegrzyn A (2007) Leptospirosis vaccines. Microb Cell Fact 6:39

32. Yanagihara Y, Kojima T, Mifuchi I (1982) Hemolytic activity of Leptospira interrogans serovar canicola cultured in protein-free medium. Microbiol Immunol 26:547-556

33. Zhang YX, Geng Y, Bi B et al (2005) Identification and classification of all potential hemolysin encoding genes and their products from Leptospira interrogans serogroup Icterohae-morrhagiae serovar lai. Acta Pharmacol Sin 26:453-461

34. Zhang YX, Geng Y, Yang JW et al (2008) Cytotoxic activity and probable apoptotic effect of Sph2, a sphigomyelinase hemolysin from Leptospira interrogans strain Lai. BMB Rep 41:119-125 